\title{
Structural and Mechanical Properties of Nanostructured C-Ag Thin Films Synthesized by Thermionic Vacuum Arc Method
}

\author{
Rodica Vladoiu, ${ }^{1}$ Aurelia Mandes $\mathbb{D}^{1},{ }^{1}$ Virginia Dinca-Balan, ${ }^{1}$ and Vilma Bursikova ${ }^{2}$ \\ ${ }^{1}$ Department of Plasma Physics, Faculty of Applied Sciences and Engineering, Ovidius University, Mamaia 124, \\ 900527 Constanţa, Romania \\ ${ }^{2}$ Department of Physical Electronics, Faculty of Science, Masaryk University, Kotlarska 2, 61137 Brno, Czech Republic
}

Correspondence should be addressed to Aurelia Mandes; amandes@univ-ovidius.ro

Received 25 October 2017; Revised 10 January 2018; Accepted 31 January 2018; Published 27 February 2018

Academic Editor: Domenico Acierno

Copyright (C) 2018 Rodica Vladoiu et al. This is an open access article distributed under the Creative Commons Attribution License, which permits unrestricted use, distribution, and reproduction in any medium, provided the original work is properly cited.

\begin{abstract}
Nanostructured C-Ag thin films of $200 \mathrm{~nm}$ thickness were successfully synthesized by the Thermionic Vacuum Arc (TVA) method. The influence of different substrates (glass, silicon wafers, and stainless steel) on the microstructure, morphology, and mechanical properties of nanostructured C-Ag thin films was characterized by High-Resolution Transmission Electron Microscopy (HRTEM), Scanning Electron Microscopy (SEM), Atomic Force Microscopy (AFM), and TI 950 (Hysitron) nanoindenter equipped with Berkovich indenter, respectively. The film's hardness deposited on glass $\left(H_{\mathrm{C}-\mathrm{Ag} / \mathrm{Gl}}=1.8 \mathrm{GPa}\right)$ was slightly lower than in the case of the $\mathrm{C}-\mathrm{Ag}$ film deposited on a silicon substrate $\left(H_{\mathrm{C}-\mathrm{Ag} / \mathrm{Si}}=2.2 \mathrm{GPa}\right)$. Also the apparent elastic modulus $E_{\text {eff }}$ was lower for C-Ag/Gl sample $\left(E_{\text {eff }}=100 \mathrm{GPa}\right)$ than for C-Ag/Si $\left(E_{\text {eff }}=170 \mathrm{GPa}\right)$, while the values for average roughness are $R_{a}=2.9 \mathrm{~nm}(\mathrm{C}-\mathrm{Ag} / \mathrm{Si})$ and $R_{a}=10.6$ $(\mathrm{C}-\mathrm{Ag} / \mathrm{Gl})$. Using the modulus mapping mode, spontaneous and indentation-induced aggregation of the silver nanoparticles was observed for both C-Ag/Gl and C-Ag/Si samples. The nanocomposite C-Ag film exhibited not only higher hardness and effective elastic modulus, but also a higher fracture resistance toughness to the silicon substrate compared to the glass substrate.
\end{abstract}

\section{Introduction}

There is great interest in applying DLC (Diamond-like carbon) films to the sliding machinery components, which is unfortunately limited by the high compressive stresses inherited and induced by the $\mathrm{sp}^{3}$ carbon bonding films as one of the major drawbacks during the synthesis of DLC films. Due to the microstructure of the films comprising nanocrystalline grains in an amorphous carbon matrix, metal containing DLC films have been shown to improve the adhesion and hardness as well as wear properties $[1,2]$.

As a soft and ductile element, Ag, embedded into the amorphous carbon matrix, may improve tribological properties like lowering brittleness rates [3], as well as mechanical properties. The excellent properties of the Ag-incorporated carbon films can be attributed to the following factors: for one thing, because of the face-centered cubic structure, Ag nanoparticle has a significantly smaller elastic modulus than carbon, and it may absorb compressive stress from the carbon matrix. Meanwhile, the toughness of composite film may be increased and the formation of bonds between the nanocrystallites and the matrix may be diminished [4-6].

Furthermore, the C-Ag thin films are of great interest, thanks to the prospect of various applications such as catalysts, optical filters, photographic processes for biology and medicine $[7,8]$. The incorporation of silver into carbon films offers the possibility of providing coatings that are hemocompatible and antibacterial [9-12]. Also, it is already known that the antibacterial activity of silver is size-dependent [13]. Another critical issue to focus on in the case of C-Ag films is the film stress, which can lead to film fracture or delamination of the film from the substrate.

In our previous works, we have reported on the TVA method used for embedding $\mathrm{Ti}, \mathrm{Al}, \mathrm{W}$, and $\mathrm{Si}$, or a combination of them into DLC films on the composition, structure, and valuable possible applications [14-18]. In this study, we discuss the influence of silver in carbon matrix in terms of mechanical properties, as well as the influence of process 


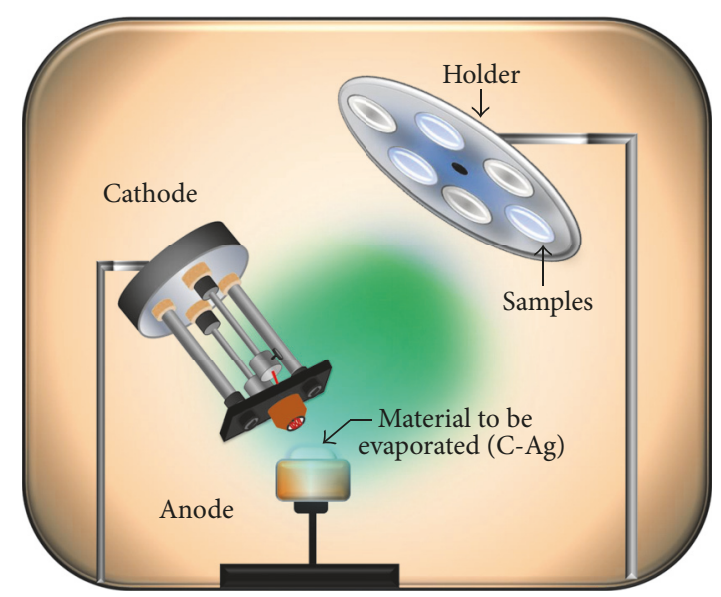

FIGURE 1: Experimental set-up for TVA single-gun configuration.

parameters on the grain size of Ag films prepared by TVA technology deposited on different substrates. Nevertheless, the effect of surface chemistry and topography can affect the nanostructure and the properties of thin films; therefore, the relevance of such a change with respect to the effect of the nature of the substrate has been analyzed below by comparing these effects in the synthesis of C-Ag films by TVA technology.

\section{Experimental Set-Up}

TVA technology is an electron beam heated cathode arc, which can be ignited and maintained in the vapors of the anode material, in vacuum conditions. The anode is a crucible, which contains the material to be evaporated (carbon powder with a purity of $99.9 \%$ and silver grains with a purity of $99.999 \%)$. The anode is kept at a high positive voltage.

2.1. Deposition Details. C-Ag nanocomposites thin films were prepared on borosilicate thin glass with a thickness of $1.2 \mathrm{~mm}$ and one side polished c-Si (crystalline silicon) substrates by the Thermionic Vacuum Arc method in a single-gun configuration (Figure 1). Also, special steel with great interest for industrial application (OLC 45-meaning stainless steel with $0.45 \%$ of carbon) discs with $15 \mathrm{~mm}$ in diameter was used as substrates. Details of the TVA apparatus used in this study are described elsewhere [19].

The cleaning process is fundamental to obtain good adhesion between the substrate and the coating and therefore good mechanical performance. For this reason, the preparation of the samples is worth a brief mention: glass and silicon substrates with the size of $1 \times 1 \mathrm{~cm}^{2}$, and OLC 45 discs, respectively, were cleaned in an ultrasonic bath with a highly effective special cleaner (ultrasonol) and then rinsed with technical grade acetone to achieve rapid drying.

The substrates were mounted on the holder and loaded into the preparation chamber above the plasma. The residual pressure of the chamber was lower than $4 \times 10^{-5} \mathrm{~Pa}$ prior to heating the filament. The cathode system was preheated approximately $45 \mathrm{~min}$ by the heat from the filament using the current of If $=45-50 \mathrm{~A}$. During this time, the outgassing continues and the residual pressure decreased again. Then an adjustable high-voltage from the current-controlled DC power supply was applied between the cathode and anode starting from 0 up to $4600 \mathrm{~V}$. The metal vapors are ionized by the same fast electrons accelerated by the anode. After the ignition of the plasma, the mobile shutter was removed, allowing homogeneous thickness and composition of the growing films on the substrates over an area of $10 \mathrm{~cm}^{2}$. Under certain operating electrical parameters, the plasma becomes stable and can be maintained for as long as the anode material is present. The time for deposition of C-Ag thin films was about 3 minutes. During the deposition, the substrate holder was not rotated. The substrate was kept at ground potential during the deposition of the plasma [20].

2.2. Structure and Morphology Investigation Details. Investigation of the films' microstructure was carried out using Transmission Electron Microscopy (TEM) performed on a Phillips CM 120 ST (acceleration voltage of $120 \mathrm{kV}$ ) TEM with a resolution point of $1.4 \AA$ and a magnification of 1.2 million times. For TEM investigation, the samples were prepared using a diamond knife to scratch the surface of the film, with alcohol as a dispersive medium and a $400 \mathrm{Cu}$ grid covered by formvar film as a holder for the sample [21].

The sample morphology was evaluated from BFTEM (Bright Field TEM) images and the mean diameter was estimated from experimental data assuming a lognormal distribution for Feret diameters [22]. Structural features of the sample were identified from the electron diffraction pattern, using the mathematical method to index the diffraction pattern and the Cohen method with modified NielsonRiley function to make an accurate determination of lattice parameters [23]. Peak positions and widths were determined using ELD module from CRISP2 application [24, 25]. All images were acquired using iTEM platform from SoftImaging System connected to MegaView III CCD camera, mounted on Philips CM120ST microscope [23]. 
Scanning Electron Microscopy (SEM) images have been performed using a Zeiss EVO 50 SEM having $\mathrm{LaB}_{6}$ cathode with Bruker EDX system. EDX measurements were carried out with a Bruker accessory fitted on the Zeiss Evo 50 scanning electron microscope. The take-off angle is $35^{\circ}$ and the detector's resolution is $133 \mathrm{eV}$.

The Atomic Force Microscopy (AFM) investigations in air have been carried out by the multifunctional device NTEGRA Prima (NT-MD Spectrum Instruments) with high precision and resolution with high-frequency (up to $5 \mathrm{MHz}$ ) AFM modes, giving information about the topography and the roughness of the thin films.

2.3. Mechanical Investigation Details. TI 950 (Hysitron) nanoindenter equipped with Berkovich indenter was used to measure nanomechanical properties of C-Ag coatings. The load resolution of the instrument is $1 \mathrm{nN}$ and load noise floor is less than $30 \mathrm{nN}$. The displacement resolution was better than $0.02 \mathrm{~nm}$ and the displacement noise floor was less than $0.2 \mathrm{~nm}$. The thermal drift was less than $0.05 \mathrm{~nm} / \mathrm{sec}$. The samples were measured under quasistatic and dynamic indentation testing conditions. Moreover, quasistatic indentations with 20 unloading segments were applied from $500 \mu \mathrm{N}$ up to the maximal load of $10 \mathrm{mN}$ in order to study the depth profile of the samples. The corresponding contact depths were in the range from about 40 to $230 \mathrm{~nm}$, depending on the sample's resistance to indentation. The indentation hardness $H_{\mathrm{IT}}$ and the reduced, effective elastic modulus $E_{r}$ were calculated according to the method of Oliver and Pharr [26]. From the reduced elastic modulus $E_{r}$, where

$$
\begin{gathered}
E_{r}=\frac{S \cdot \sqrt{\pi}}{2 \cdot \beta \cdot \sqrt{A_{p}}}, \\
S=\left(\frac{d F}{d h}\right)_{h_{\max }},
\end{gathered}
$$

it was possible to calculate the elastic modulus $\left(E_{\text {eff }}\right)$ according to the following equation:

$$
E_{\mathrm{eff}}=\frac{1}{1 / E_{r}-\left(1-v_{i}^{2}\right) / E_{i}}=\frac{E_{s}}{1-v_{s}^{2}} .
$$

Here $\beta$ is a geometrical constant, which is 1.034 for the Berkovich indenter, $A_{p}$ is the projected contact area. $S$ is the so-called unloading stiffness calculated for maximum indentation depth $h_{\max } ; F$ is the loading force and $h$ is the indentation depth reached at the given loading force; $E_{i}$ is the Young's modulus and $v_{i}$ is the Poisson ratio of the diamond indenter and $E_{s}$ is the Young's modulus and $\nu_{s}$ is the Poisson ratio of the studied sample.

The calibration of the transducer for quasistatic and nanodynamic mechanical analysis (nanoDMA) was done before each set of measurement in quasistatic as well as dynamic mode. The electrostatic force versus displacement calibration curve remained linear in the whole given displacement range. The fitting error was under $1 \%$ in the whole range; ordinarily, it was around $0,5 \%$. The indenter tip calibration was done on fused silica sample for several different indentation depths with contact depths larger than $1 / 3$ of the tip radius. Before the indentation tests the sample surface was scanned using the in situ scanning probe (SPM) possibility. The places with the minimum roughness were chosen for the nanoindentation tests in order to minimize the effect of the roughness on the indentation data.

The indentation-induced cracking or delamination at relatively high indentation loads $(0.1$ to $0.3 \mathrm{~N})$ was used to study qualitatively the fracture resistance and the adhesion of the films to the substrates. The thickness of the deposited films was around $200 \mathrm{~nm}$, measured by means of a profilometer.

\section{Results and Discussions}

3.1. Microstructure and Morphology of C-Ag Films. Figure 2 shows the Bright Field Transmission Electron Microscopy (BFTEM) and the High-Resolution Transmission Electron Microscopy (HRTEM) images of the film edges for C-Ag sample on silicon ( $\mathrm{Si}$ ), glass (Gl), and stainless steel (OLC) substrates. The samples of film-substrate systems were denominated as C-Ag/Si, C-Ag/Gl, and C-Ag/OLC. Performing the image' processing feature in analysis iTEM platform, one can select and measure Feret diameters of $100 \mathrm{Ag}$ nanoparticles. Moreover, an experimental histogram has been carried out by using the SciDAVIS program, fitted by lognormal function [27]. Interference fringes associated with graphitized carbon (C: $d=0.34 \mathrm{~nm})$ and Ag phase $(d=0.24 \mathrm{~nm})$ could be identified.

The histograms of grain size are revealed in Figure 3. Grain size distribution was built using the projected area estimated from TEM images. That is not necessarily crystallite size, as the grain can be polycrystalline. The Scherrer formula applied to electron diffraction shows lower values for the crystallite size of each sample (C-Ag/Si $=23.2 \mathrm{~nm}, \mathrm{C}-\mathrm{Ag} / \mathrm{glass}$ $=6.85 \mathrm{~nm}$, and C-Ag/OLC $=15.99 \mathrm{~nm})$.

The electron diffraction was carried out at $880 \mathrm{~mm}$ camera length. The background was fitted with 9th-degree polynomial function. The results were saved as ASCII files and used to index diffraction pattern and to evaluate the lattice parameters. Figure 4 presents a snapshot of CRISP2 integrated profile calculated from diffraction pattern for $\mathrm{C}-\mathrm{Ag} / \mathrm{Si}$ sample (a), C-Ag/Gl (b), and C-Ag/OLC (c), respectively. The intensity (in arbitrary units - u.a.) is depicted on $y$-axis and the radius, in pixels, on $x$-axis. The results are converted in degrees and angstroms in text file saved by the CRISP2 using user camera constant (in our case $44.56 \mathrm{~nm} *$ pixel). The "odd" numbers are calculated by CRISP2 based on radius of selected circle from diffraction pattern and maximum of intensity (for each axis 10 numbers are shown. For instance, in the case of $\mathrm{C}$ - $\mathrm{Ag} / \mathrm{Si}$ on $x$-axis, there is $523 / 9=58.11$ and for $y$-axis $216.6 / 9=24.07$ ).

The structure of $\mathrm{C}-\mathrm{Ag} / \mathrm{Si}$ exhibits the $\mathrm{Si}$ and $\mathrm{Ag}$ phases, but also some oxide phase. The lattice parameter was $0.414 \pm$ $0.005 \mathrm{~nm}$ using the analytical method and $0.414 \pm 0.002 \mathrm{~nm}$ using the Cohen method. The relative error was $1.29 \%$ in both cases according to [28].

The results for C-Ag/Gl sample revealed a complex material with large partial carbon (onion-like carbon with a 

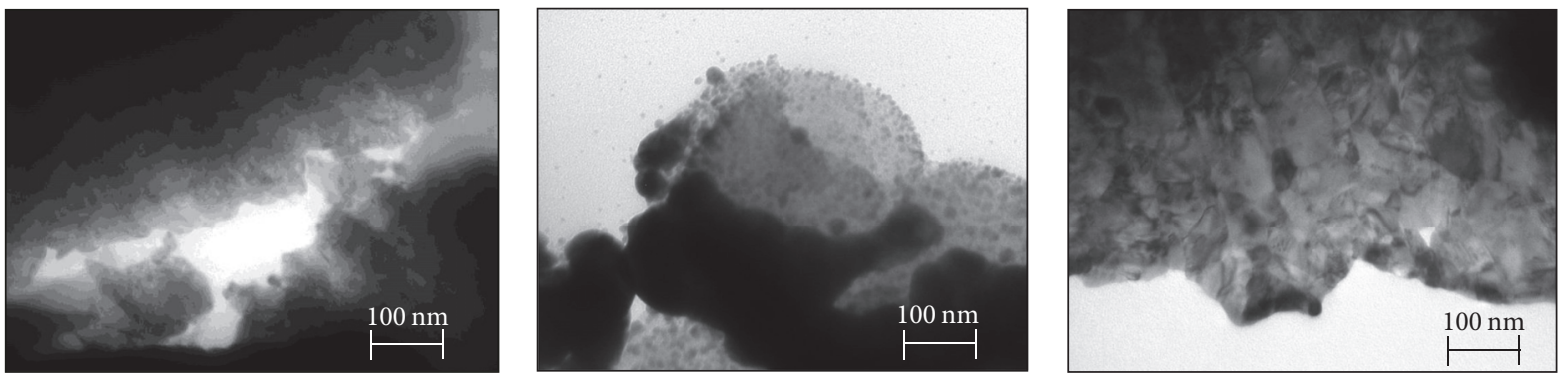

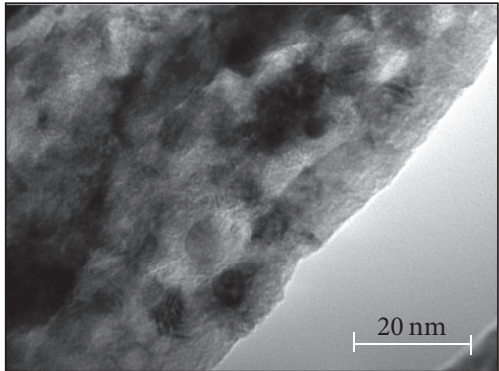

(a) $\mathrm{C}-\mathrm{Ag} / \mathrm{Si}$

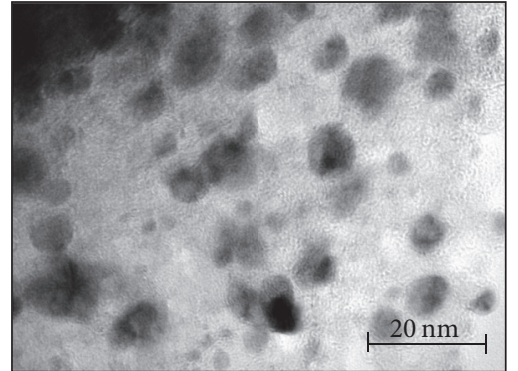

(b) $\mathrm{C}-\mathrm{Ag} / \mathrm{Gl}$

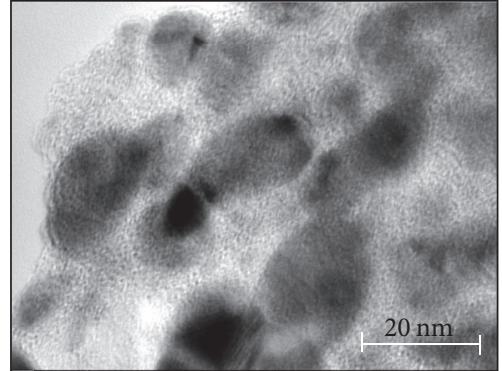

(c) $\mathrm{C}-\mathrm{Ag} / \mathrm{OLC}$

Figure 2: BFTEM images (top) and HRTEM at $20 \mathrm{~nm}$ scale (bottom) for C-Ag samples.

diameter of $160 \mathrm{~nm}$ ) covered by small crystalline Ag particles $(7 \mathrm{~nm})$. The lattice parameter for the Ag phase in this case was $0.412 \pm 0.003 \mathrm{~nm}$ calculated from the analytical method and $0.406 \pm 0.003 \mathrm{~nm}$ using the Cohen method (relative errors: $0.89 \%$ and $0.66 \%$, resp.).

For sample C-Ag/OLC, only Ag phase has been found. The lattice parameter was calculated as $0.411 \pm 0.002 \mathrm{~nm}$ by the analytical method and $0.408 \pm 0.001 \mathrm{~nm}$ using the Cohen method, with relative errors of $0.6 \%$ and $0.02 \%$, respectively, compared with data from [29]. In addition, very weak peaks $(1,3,5,7)$ have been found which might be attributed to $\mathrm{Ag}_{2} \mathrm{O}$ phase.

The characterization based on the SEM images is presented in Figure 5 (image size $1000 \times 750$, Mag 15000x). The mapping (image size $750 \times 563$, Mag $15000 x$ ) reveals that the films exhibit a relatively smooth surface morphology. Based on the SEM images, the equipment can generate characteristic X-ray as well as secondary and back-scattered electrons (pulse height $0.95 \mathrm{kcps})$. Only three peaks could be identified: Ag (29\%), C (deposited as a thin film), and Si (due to the substrate), in a very good agreement with the TEM results, proving the high purity of the deposition without any other contamination.

The topography of the samples as well as the roughness of the films were investigated by AFM images (Figure 6). The average roughness of the sample CAg/Si was $R_{a}=2.9 \mathrm{~nm}$ and the root mean square roughness $R_{q}=6.5 \mathrm{~nm}$. In the case of C-Ag/Gl, the roughness was higher: $R_{a}=10.6 \mathrm{~nm}$ and $R_{q}=$ $18.1 \mathrm{~nm}$.

3.2. Mechanical Properties of C-Ag Films. The indentation resistance of the C-Ag films deposited on glass and silicon substrates was studied using several different indentation techniques. The first set of measurements was carried out using quasistatic nanoindentations with 20 unloading segments. The time dependence of the indentation load and an example of the resulting displacement obtained during indentation testing of C-Ag film deposited on silicon substrate are illustrated in Figure 7.

The quasistatic loading curves with 20 unloading segments shown in Figure 8 were obtained at eight different places on the C-Ag film deposited on a single crystalline silicon substrate. Each color represents an individual measurement at a different place on the sample. The curves obtained from different places show only a slight scatter. Each of the unloading segments was analyzed according to Oliver and Pharr approach in order to get dependence of the hardness and reduced elastic modulus from the near surface of the C-Ag film up to the film-substrate interface. The last (20th) unloading segments in Figure 8 exhibit an elbow, which is characteristic of unloading response of single crystalline silicon, and it is formed due to pressureinduced phase transformation. According to this effect, it is possible to recognize when the influence of the substrate on the measured nanoindentation data starts to predominate.

Similar curves were obtained on a glass substrate; the depth profiles of hardness and effective elastic modulus obtained at different places are shown for $\mathrm{C}-\mathrm{Ag} / \mathrm{Si}$ and $\mathrm{C}$ $\mathrm{Ag} / \mathrm{Gl}$ samples in Figure 9. According to these results, it is possible to confirm that the data are well reproducible for the samples set.

Measurements at different places on the sample are distinguished with a different color.

The comparison of the average hardness and effective modulus values dependent on the indentation depth is 

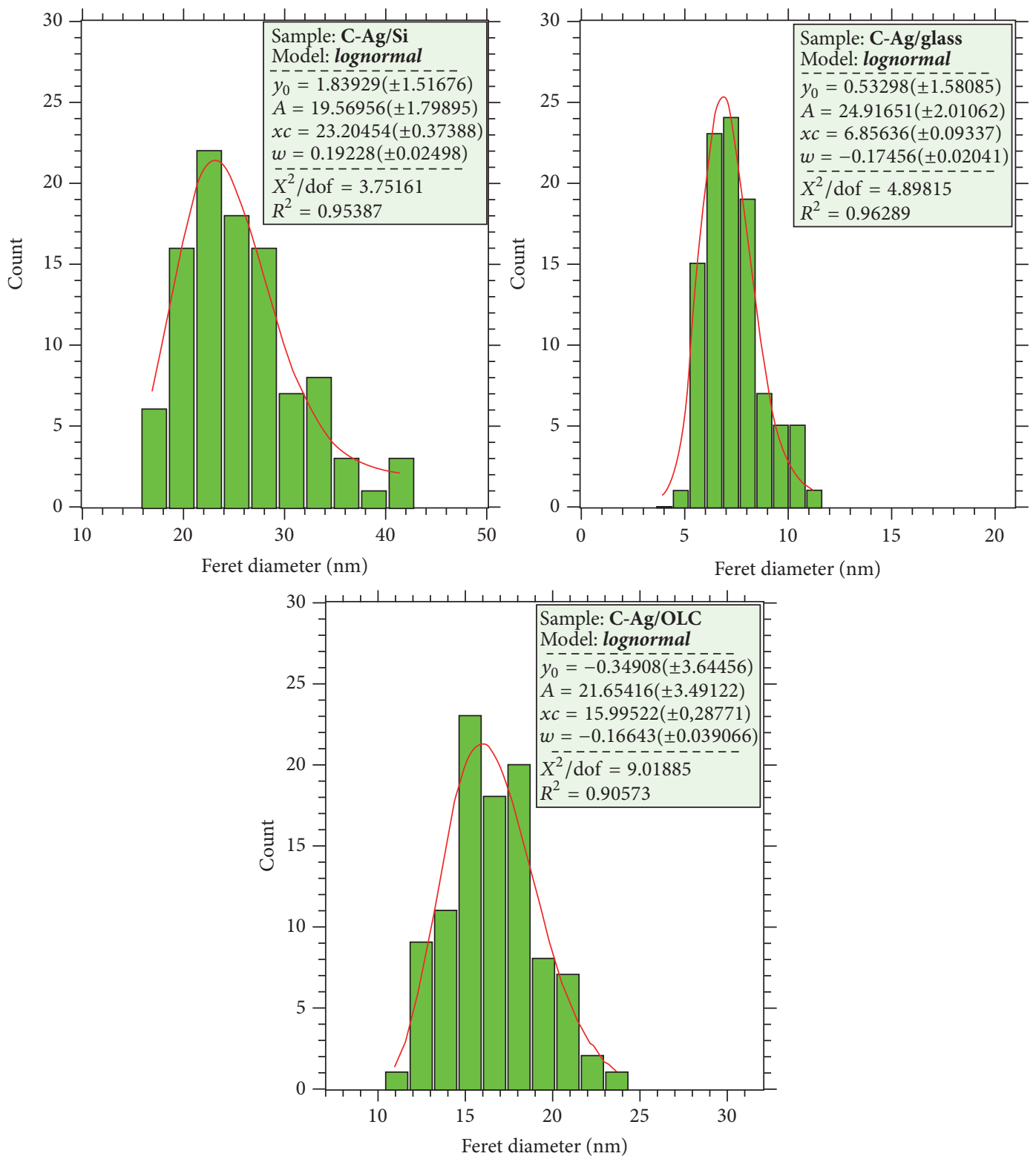

Figure 3: Grain size distributions of the C-Ag deposited on silicon, on glass and on OLC substrate.

presented in Figure 10. It is shown that the average hardness values gradually increase with the contact depth $h_{c}$. The hardness results approach 2 and $1.8 \mathrm{GPa}$ for contact depths below $40 \mathrm{~nm}$ as it can be seen in Figure 10. Although the structures of $\mathrm{Ag} / \mathrm{Si}$ and $\mathrm{C}-\mathrm{Ag} / \mathrm{Gl}$ samples are very similar, there is, at first sight, a large difference in the effective elastic modulus profile for samples deposited on silicon and glass substrates. In the case of $\mathrm{C}-\mathrm{Ag} / \mathrm{Si}$, there is a drop in elastic modulus values at about $155 \mathrm{~nm}$, suggesting the start of the substrate influence. The hardness of silicon substrate was $11 \pm 1 \mathrm{GPa}$, and the hardness of glass sample was $7 \pm 1 \mathrm{GPa}$. The measured hardness of both C-Ag films is much lower than that of the silicon and glass substrates. In the case of the soft films on the hard substrates, the measured hardness started to be influenced significantly by the substrate in indentation depths approaching half of the film thickness, which is $100 \mathrm{~nm}$. Because of the gradient character of the films, the hardness increase is starting earlier, so it is difficult to determine the depth, where the substrate influence really starts. However, the influence of the substrate elastic modulus should starts at much lower values than $100 \mathrm{~nm}$. Thus, the effective elastic modulus of $\mathrm{C}-\mathrm{Ag} / \mathrm{Si}$ sample appears as high as $180 \mathrm{GPa}$ because the elastic modulus data for the depth around $40 \mathrm{~nm}$ is fully influenced by the silicon substrate effective modulus, which for low loads is also around $180 \mathrm{GPa}$. The drop in $E_{\text {eff }}$ values at about $160 \mathrm{~nm}$ was probably caused by the well-known pressure-induced transformation of the single crystalline silicon.

The fracture resistance and the resistance against delamination were studied qualitatively using optical microscopy 

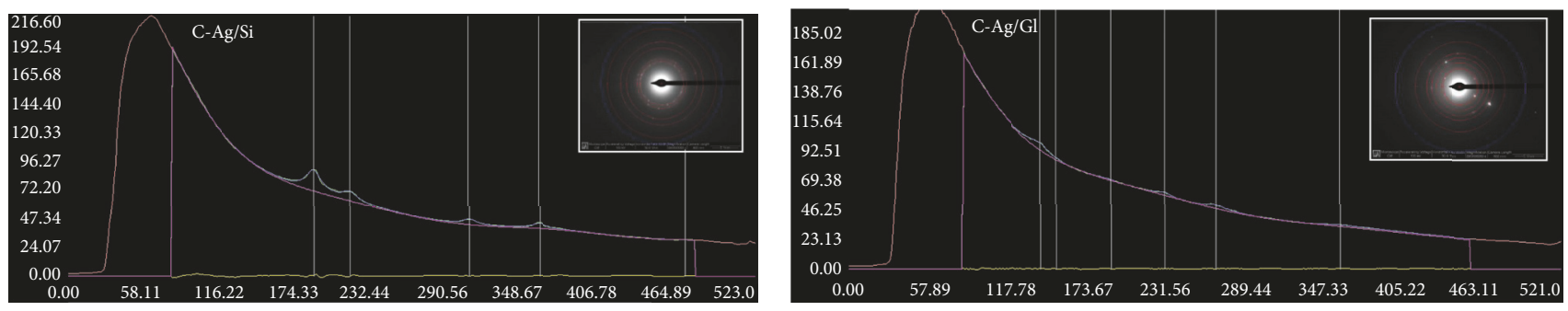

(a)

(b)

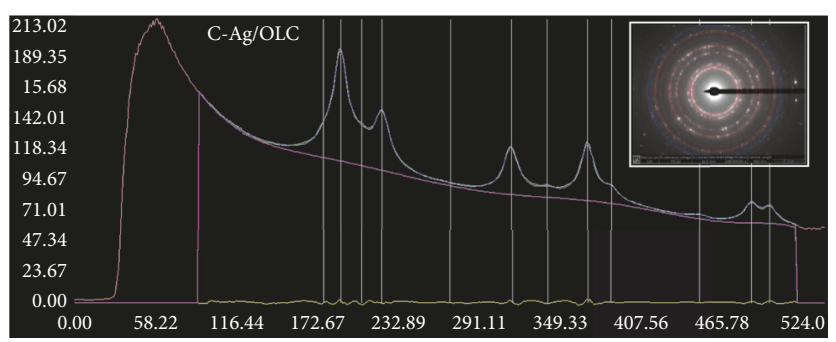

(c)

FIGURE 4: Integrated diffraction profile for different types of substrates. Inset: diffraction patterns.
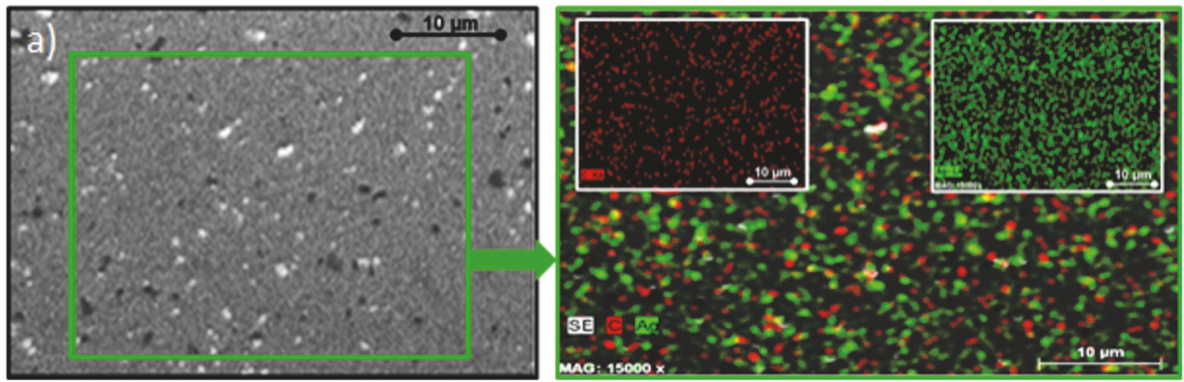

(a)

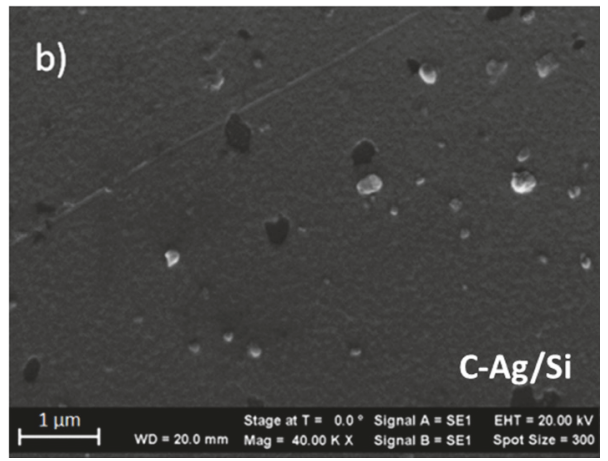

(b)

FIgURE 5: SEM image with mapping (a) and picture (b) of the C- $\mathrm{Ag} / \mathrm{Si}$.

observation of the indentation prints carried out at relatively large loads $(0.1-0.3 \mathrm{~N})$. The C-Ag sample on silicon showed much better resistance against indentationinduced cracking and delamination. The C-Ag sample on glass started to crack and delaminate around the indentation prints earlier comparing to the film on the silicon substrate.
The different behavior was probably caused by different internal stresses (due to different elastic mismatch at the interface).

The elastic modulus values were measured by a nanodynamical mechanical analysis using the modulus mapping capability. During the in situ imaging process, the 


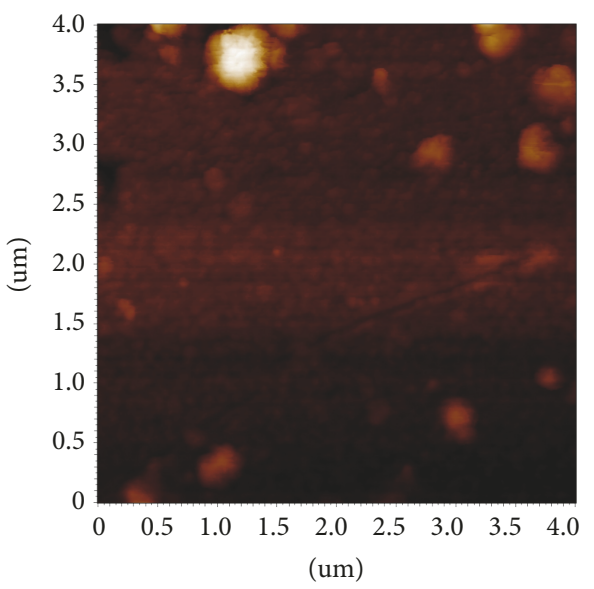

(a)

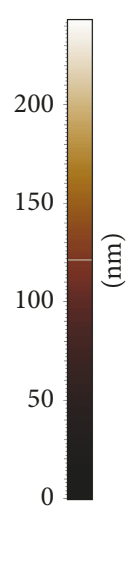

FIGURE 6: AFM 2D and 3D surface topography image of C-Ag/Gl sample.

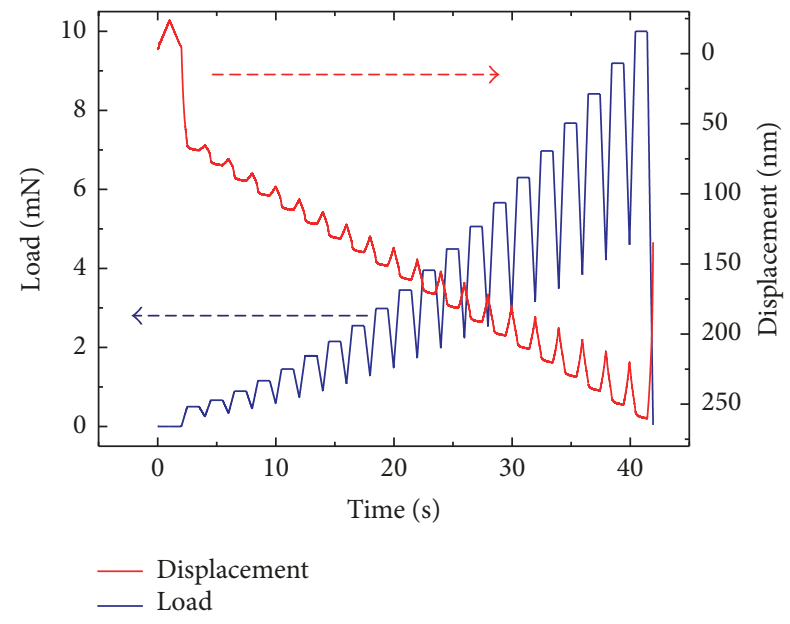

FIgURE 7: Time dependence of the load during the quasistatic nanoindentation test with 20 unloading segments and an example of the resulting displacement carried out on $\mathrm{C}-\mathrm{Ag} / \mathrm{Si}$ sample.

system continuously monitors the dynamic response of the sample to the oscillating load as a function of the position.

Therefore, at each image pixel $(256 \times 256$ pixels $)$, the storage and loss stiffness and the storage and loss moduli values are determined. Quantitative maps of the storage stiffness and the storage modulus (Figure 11) were carried out at a frequency of $300 \mathrm{~Hz}$ with Cube Corner indenter. The oscillating force amplitude was $1 \mu \mathrm{N}$, the oscillation frequency was $300 \mathrm{~Hz}$ and the displacement amplitude was around $1 \mathrm{~nm}$. The results confirmed our previous assumptions about the influence of the silicon substrate on the measured data. It was shown that the modulus values are similar to those obtained on a glass substrate. Moreover, it was possible to visualize the local changes in the modulus and stiffness showing that the coatings contain Ag particle aggregates with elastic response different from the matrix.

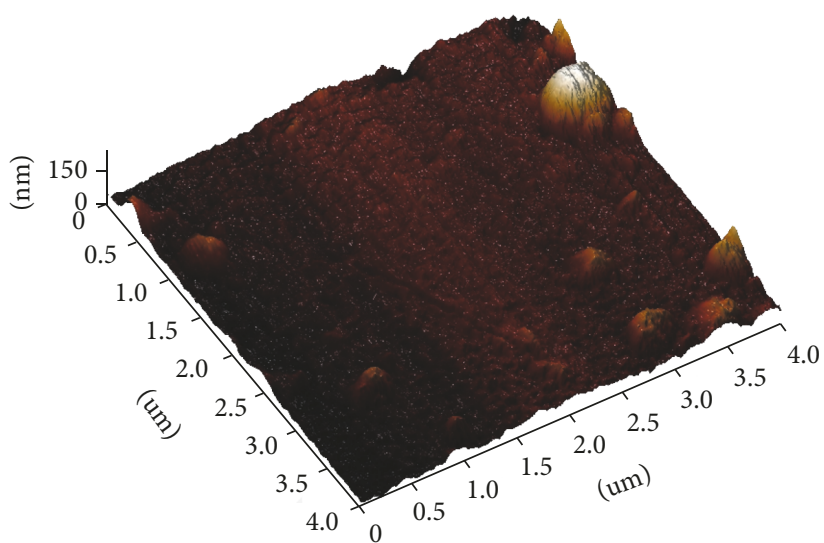

(b) 


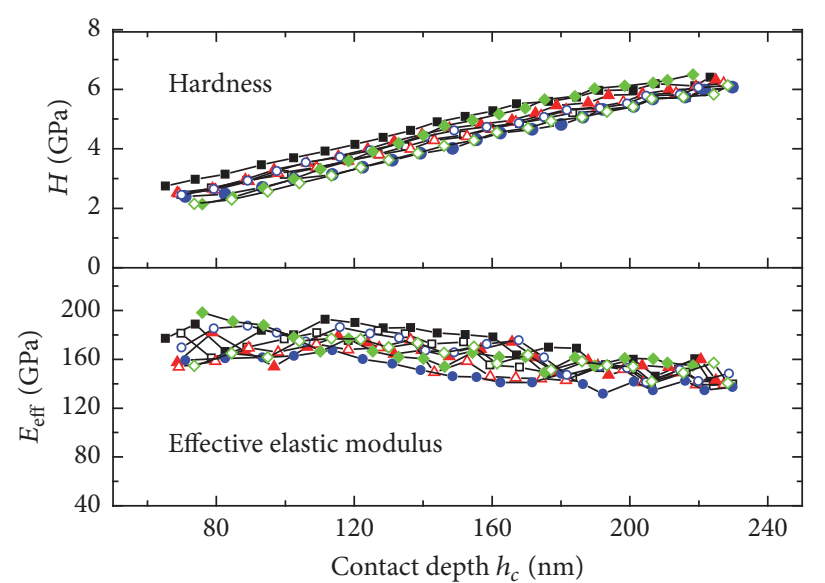

(a)

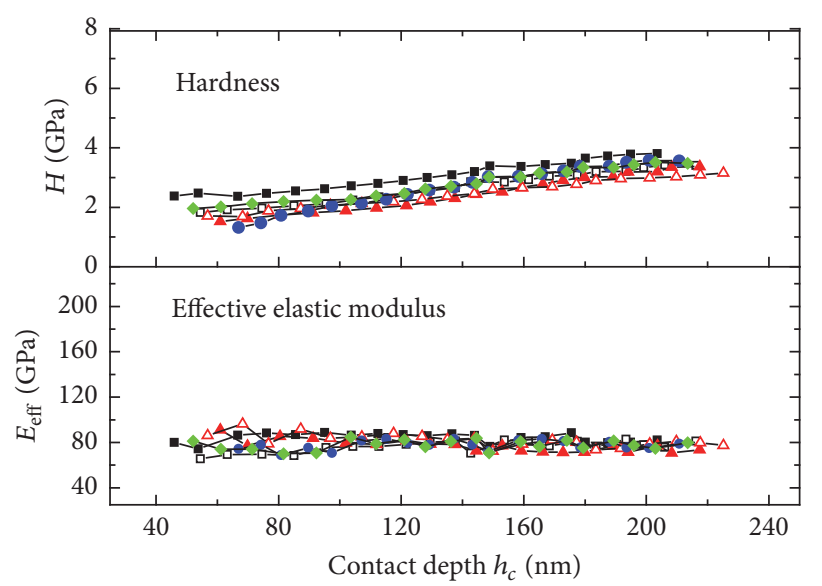

(b)

Figure 9: Dependence of the hardness and effective elastic modulus (calculated according to (2)) on contact depth, $\mathrm{C}$ - $\mathrm{Ag} / \mathrm{Si}$ (a) and $\mathrm{C}$ - $\mathrm{Ag} / \mathrm{Gl}$ (b).

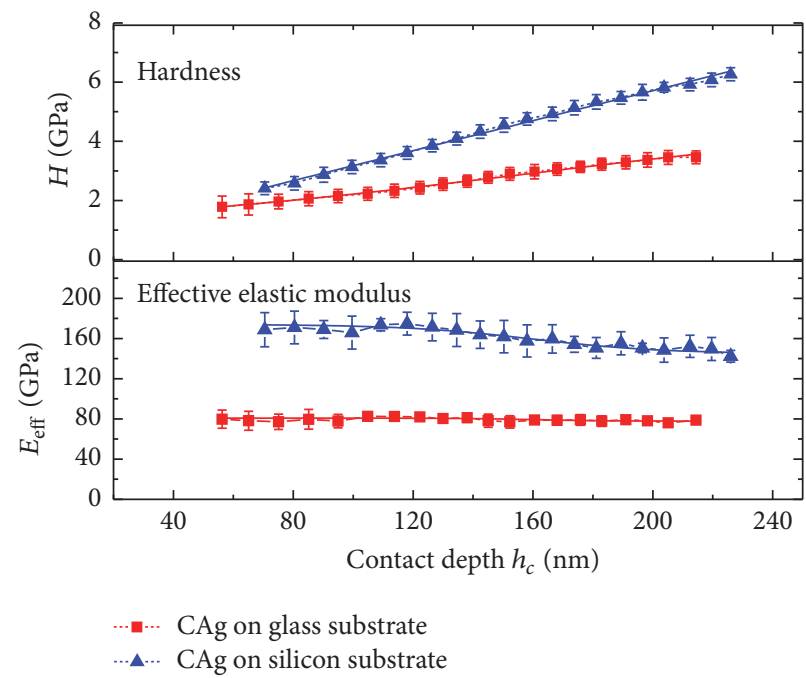

FIGURE 10: The comparison of the averaged hardness and effective modulus values depending on the indentation depth.
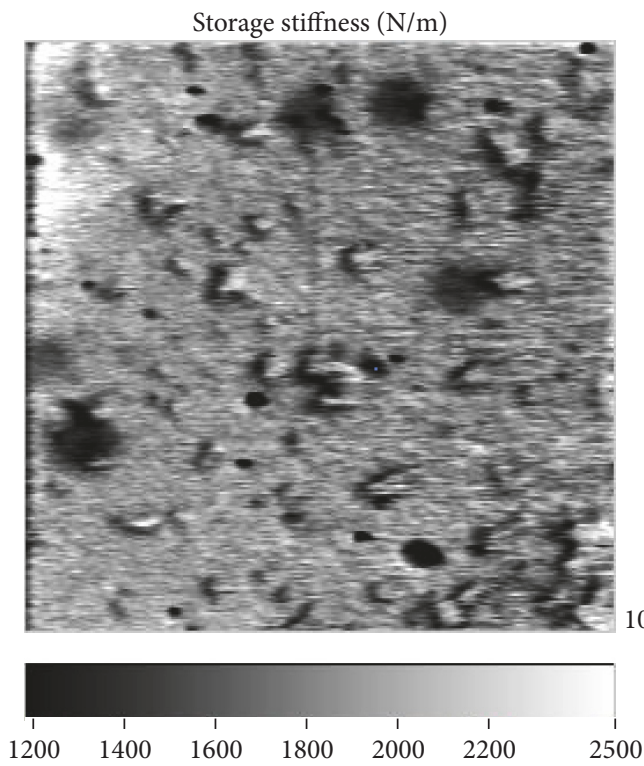

(a)
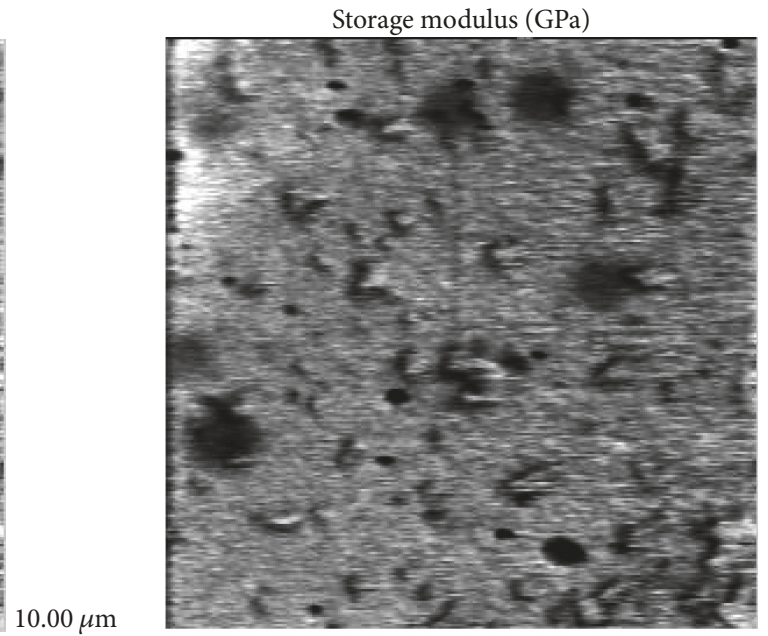

$10.00 \mu \mathrm{m}$

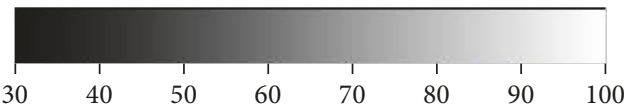

(b)

Figure 11: Quantitative maps of the storage stiffness (a) and the storage modulus (b) obtained on C-Ag/Si surface. 
Ag nanocrystallites dispersed in the DLC matrix. Within a certain range of the Ag content and the nanograin size, the intrinsic compressive stress was reduced, while its hardness was improved. In this case, no delamination or fracture of the films was noticed.

In particular, Ag-DLC film deposited on silicon exhibited slightly higher hardness and similar elastic modulus and lower intrinsic stress compared with the films deposited on glass. It was concluded that Ag aggregates on the indented surface are forming Ag layer between the indenter and the film surface, which may be responsible for the decrease in the hardness values at the film surface region in relation to the carbon coatings deposited using TVA at similar conditions.

\section{Conflicts of Interest}

The authors declare that they have no conflicts of interest.

\section{Acknowledgments}

This work was supported by a grant of the Ministry of Research and Innovation, CNDI-UEFISCDI, Project 70/2017, PN-III-P4-ID-PCE-2016-0750, within PNCDI III, and the Czech Science Foundation, Project 15-17875S.

\section{References}

[1] C. Donnet, "Recent progress on the tribology of doped diamond-like and carbon alloy coatings: A review," Surface and Coatings Technology, vol. 100-101, no. 1-3, pp. 180-186, 1998.

[2] A. A. Voevodin, S. V. Prasad, and J. S. Zabinski, "Nanocrystalline carbide/amorphous carbon composites," Journal of Applied Physics, vol. 82, no. 2, pp. 855-858, 1997.

[3] N. K. Manninen, F. Ribeiro, A. Escudeiro, T. Polcar, S. Carvalho, and A. Cavaleiro, "Influence of Ag content on mechanical and tribological behavior of DLC coatings," Surface and Coatings Technology, vol. 232, pp. 440-446, 2013.

[4] X. Yu, Y. Qin, C. B. Wang, Y. Q. Yang, and X. C. Ma, "Effects of nanocrystalline silver incorporation on sliding tribological properties of Ag-containing diamond-like carbon films in multi-ion beam assisted deposition," Vacuum, vol. 89, no. 1, pp. 82-85, 2013.

[5] H.-S. Zhang, J. L. Endrino, and A. Anders, "Comparative surface and nano-tribological characteristics of nanocomposite diamond-like carbon thin films doped by silver," Applied Surface Science, vol. 255, no. 5, pp. 2551-2556, 2008.

[6] C. Wang, X. Yu, and M. Hua, "Microstructure and mechanical properties of Ag-containing diamond-like carbon films in midfrequency dual-magnetron sputtering," Applied Surface Science, vol. 256, no. 5, pp. 1431-1435, 2009.

[7] J. Hanus, M. Drabik, P. Hlidek, H. Biederman, G. Radnoczi, and D. Slavinska, "Some remarks on Ag/C:H nanocomposite films," Vacuum, vol. 83, no. 2, pp. 454-456, 2009.

[8] H. Zoubos, L. E. Koutsokeras, D. F. Anagnostopoulos et al., "Broadband optical absorption of amorphous carbon/Ag nanocomposite films and its potential for solar harvesting applications," Solar Energy Materials \& Solar Cells, vol. 117, pp. 350-356, 2013.

[9] K. Baba, R. Hatada, S. Flege et al., "Preparation and antibacterial properties of Ag-containing diamond-like carbon films prepared by a combination of magnetron sputtering and plasma source ion implantation," Vacuum, vol. 89, no. 1, pp. 179-184, 2013.

[10] B. Jiang, C. Tian, G. Song et al., "A green route to synthesize novel Ag/C antibacterial agent," Materials Research Bulletin, vol. 47, no. 2, pp. 458-463, 2012.

[11] F. P. Schwarz, I. Hauser-Gerspach, T. Waltimo, and B. Stritzker, "Antibacterial properties of silver containing diamond like carbon coatings produced by ion induced polymer densification," Surface and Coatings Technology, vol. 205, no. 20, pp. 48504854, 2011.

[12] N. R. Srinivasan, P. A. Shankar, and R. Bandyopadhyaya, "Plasma treated activated carbon impregnated with silver nanoparticles for improved antibacterial effect in water disinfection," Carbon, vol. 57, pp. 1-10, 2013.

[13] J. R. Morones, J. L. Elechiguerra, A. Camacho et al., "The bactericidal effect of silver nanoparticles," Nanotechnology, vol. 16, no. 10, pp. 2346-2353, 2005.

[14] C. P. Lungu, I. Mustata, G. Musa et al., "Unstressed carbonmetal films deposited by thermionic vacuum arc method," Journal of Optoelectronic and Advanced Materials, vol. 8, no. 1, pp. 74-77, 2006.

[15] V. Ciupina, R. Vladoiu, A. Mandes, G. Musa, and C. P. Lungu, "TEM investigation of the C-Me multilayer nanocomposites deposited by Thermionic Vacuum Arc (TVA) method," Journal of Optoelectronic and Advanced Materials, vol. 10, no. 11, pp. 2958-2962, 2008.

[16] V. Ciupina, R. Vladoiu, C. P. Lungu et al., "Investigation of the $\mathrm{SiC}$ thin films synthetized by Thermionic Vacuum Arc method (TVA)," The European Physical Journal D, vol. 66, no. 4, article no. 99, 2012.

[17] V. Kuncser, M. Valeanu, G. Schinteie et al., "Exchange bias and spin valve systems with Fe-Mn antiferromagnetic pinning layers, obtained by the thermo-ionic vacuum arc method," Journal of Magnetism and Magnetic Materials, vol. 320, no. 14, pp. e226-e230, 2008.

[18] R. Vladoiu, V. Dinca, and G. Musa, "Surface energy evaluation of unhydrogenated DLC thin film deposited by thermionic vacuum arc (TVA) method," The European Physical Journal D, vol. 54, no. 2, pp. 433-437, 2009.

[19] R. Vladoiu, V. Ciupina, A. Mandes, V. Dinca, M. Prodan, and G. Musa, "Growth and characteristics of tantalum oxide thin films deposited using thermionic vacuum arc technology," Journal of Applied Physics, vol. 108, no. 9, Article ID 093301, 2010.

[20] G. Musa, I. Mustata, M. Blideran et al., "Thermioniv vacuum arc - New technique for high purity carbon thin film deposition," Acta Physica Slovaca, vol. 55, no. 4, pp. 417-421, 2005.

[21] V. S. Teodorescu and M.-G. Blanchin, "Fast and simple specimen preparation for TEM studies of oxide films deposited on silicon wafers," Microscopy and Microanalysis, vol. 15, no. 1, pp. 15-19, 2009.

[22] http://www.soft-imaging.net.

[23] J. I. Langford, "The accuracy of cell dimensions determined by Cohen's method of least squares and the systematic indexing of powder data," Journal of Applied Crystallography, vol. 6, no. 3, pp. 190-196, 1973.

[24] S. Hovmöller, "CRISP: crystallographic image processing on a personal computer," Ultramicroscopy, vol. 41, no. 1-3, pp. 121-135, 1992.

[25] X. D. Zou, Y. Sukharev, and S. Hovmöller, "Quantitative electron diffraction - new features in the program system ELD," Ultramicroscopy, vol. 52, no. 3-4, pp. 436-444, 1993. 
[26] W. C. Oliver and G. M. Pharr, "Measurement of hardness and elastic modulus by instrumented indentation: advances in understanding and refinements to methodology," Journal of Materials Research, vol. 19, no. 1, pp. 3-20, 2004.

[27] http://scidavis.sourceforge.net/.

[28] R. W. G. Wyckoff, Crystal Structures (WWW-MINCRYST, SILICON-4221), vol. 1, pp. 26-27, 1963.

[29] R. W. G. Wyckoff, Crystal structures (WWW-MINCRYST, SILVER-4219), vol. 1, pp. 7-10, 1963.

[30] J. Li, Y. Lin, and B. Zhao, "Spontaneous agglomeration of silver nanoparticles deposited on carbon film surface," Journal of Nanoparticle Research, vol. 4, no. 4, pp. 345-349, 2002.

[31] R. Vladoiu, V. Ciupina, C. Surdu-Bob et al., "Properties of the carbon thin films deposited by thermionic vacuum arc," Journal of Optoelectronic and Advanced Materials, vol. 9, no. 4, pp. 862866, 2007. 


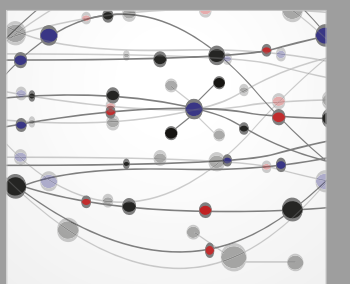

The Scientific World Journal
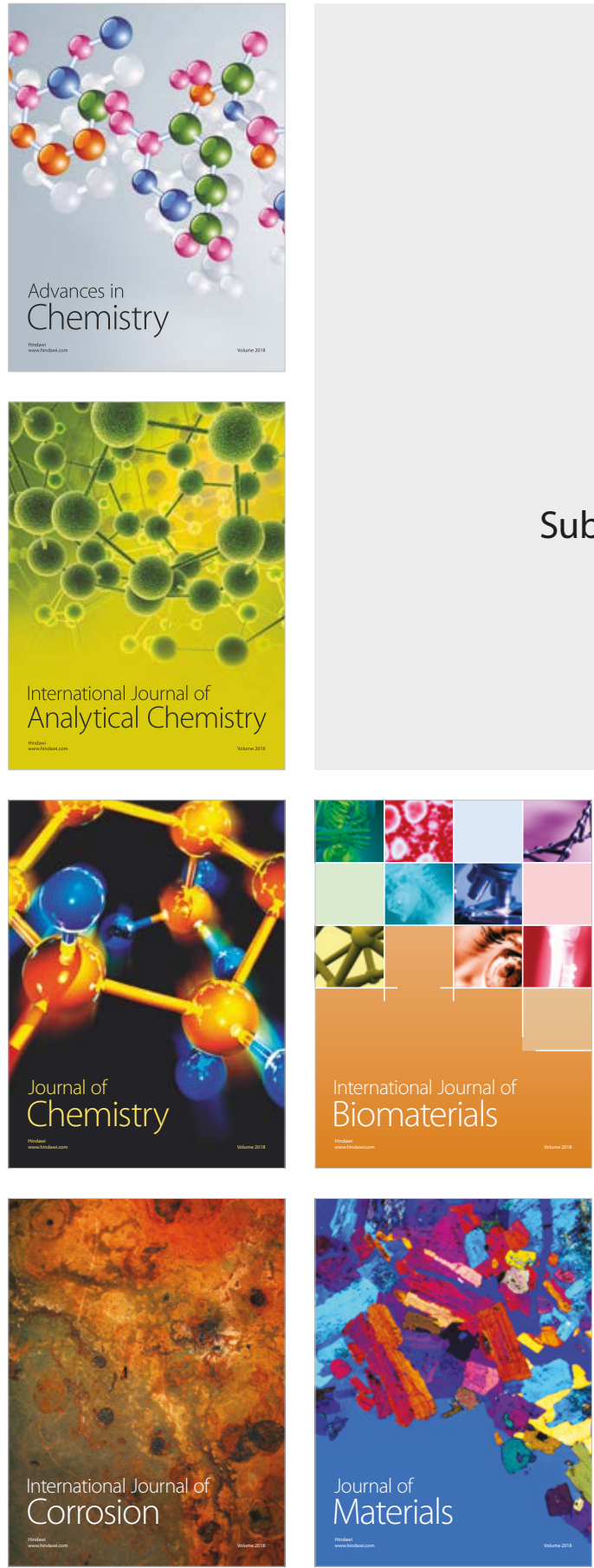

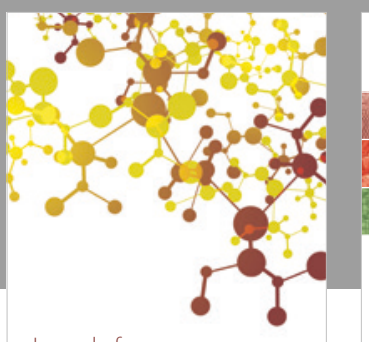

Journal of

Applied Chemistry
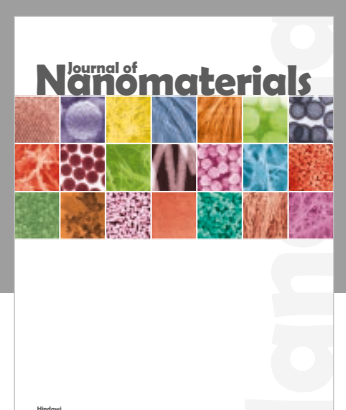

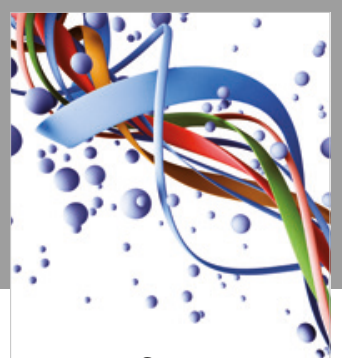

Scientifica

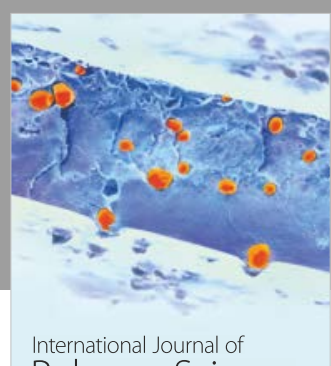

Polymer Science

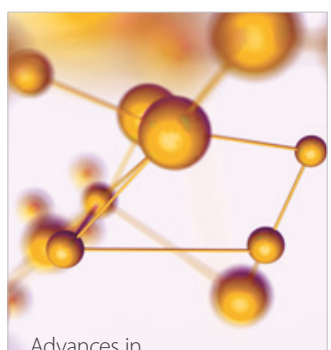

Physical Chemistry
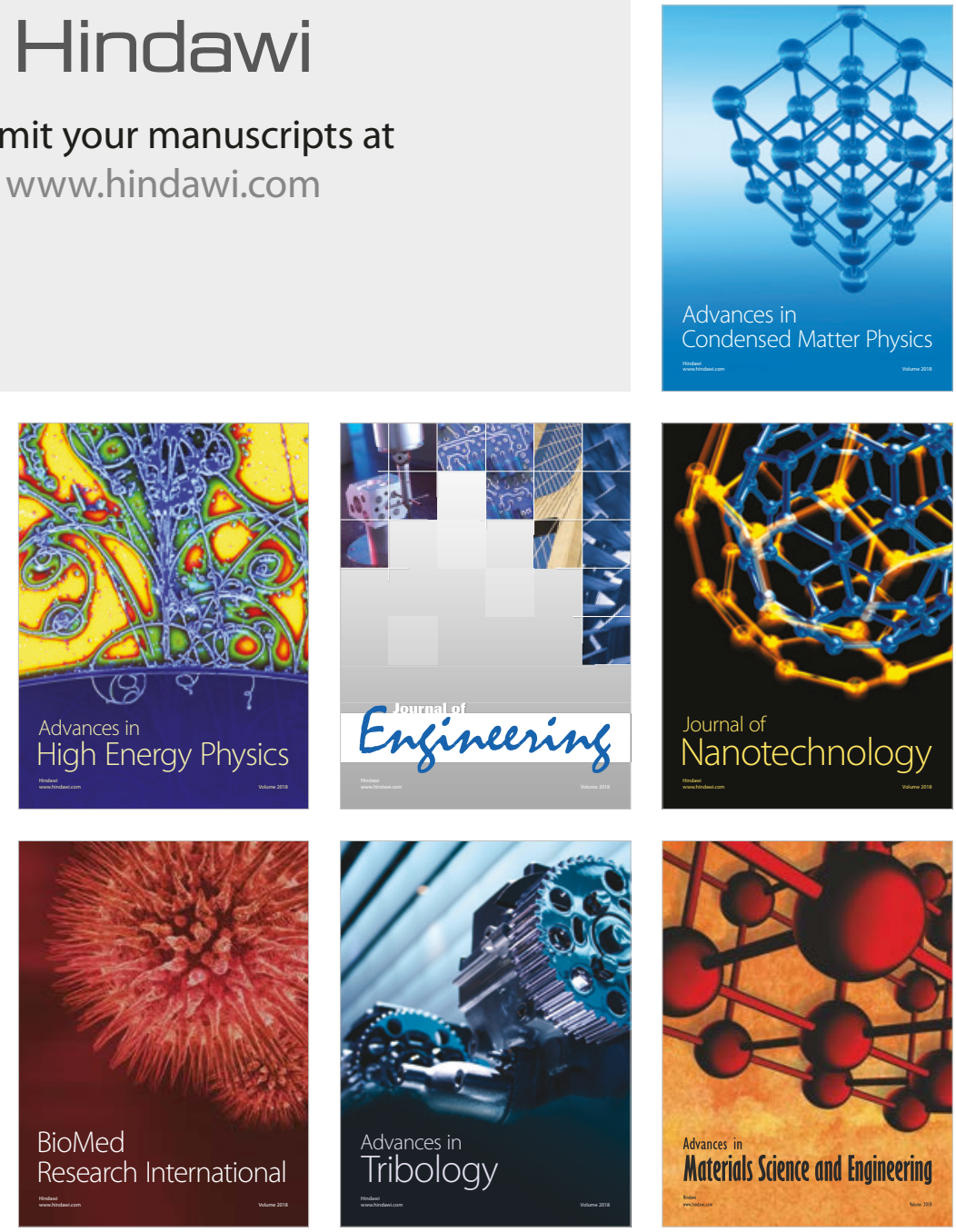